\title{
Clínica de la hipertensión arterial, para el médico general
}

\section{Clinical aspects of arterial hypertension for the general practitioner}

\author{
Armando Vidalón-Fernández ${ }^{1,2,3}$ \\ ${ }^{1}$ Doctor en Medicina, Especialista en Nefrología. \\ ${ }^{2}$ Ex Profesor Principal, Facultad de Medicina, UNMSM. \\ ${ }^{3}$ Ex Presidente Sociedad Peruana de Nefrología, Sociedad Peruana de Transplantes, Sociedad Peruana de Hipertensión Arterial.
}

\section{Resumen}

Se revisa los aspectos clínicos básicos de la hipertensión arterial (HTA). Se detalla la definición actualizada de la HTA y su clasificación, el propósito principal del diagnóstico, la correcta medición de la presión arterial (PA) y causas identificables de HTA, la evaluación clínica y de laboratorio. Se enumera los factores de riesgo vascular y daño de órgano blanco, así como la importancia del sobrepeso/obesidad y errores comunes de concepto sobre la HTA.

Palabras clave: Hipertensión, clasificación, diagnóstico; presión sanguínea; diagnóstico clínico.

\begin{abstract}
We review basic clinical aspects of arterial hypertension (AHT). A detailed and updated definition of arterial hypertension and its correspondent classification is presented, the purpose of diagnosis, correct measurement of blood pressure and AHT identifiable causes, clinical and laboratory evaluation, vascular risk factors and target organ damage, as well as the importance of overweight/obesity and common misconceptions on AHT.
\end{abstract}

Keywords: Hypertension, classification, diagnosis; blood pressure; diagnosis clinical.

\section{DEFINICIÓN}

La hipertensión arterial se define como la presión arterial sistólica (PAS) de $140 \mathrm{mmHg}$ o mayor, presión arterial diastólica (PAD) de $90 \mathrm{mmHg}$ o mayor o estar medicado con fármacos antihipertensivos $^{(1)}$.

La relación entre la presión arterial (PA) y la enfermedad cardiovascular (ECV) es continua, consistente e independiente de otros factores de riesgo ${ }^{(2)}$. Por lo tanto, la definición de HTA es numéricamente arbitraria e incluso en el rango normotensivo las personas con menos niveles de PA poseen tasas más bajas de $\mathrm{ECV}^{(3)}$.

Las guías remarcan que la decisión en disminuir la PA en un paciente en particular no se basa exclusivamente en el nivel de la PA, sino en la evaluación del riesgo total cardiovascular en tal individuo $^{(1,2)}$.

La hipertensión sistólica aislada consiste en cifras $\geq 140 \mathrm{mmHg}$ y la diastólica en $<90 \mathrm{mmHg}$. La presión del pulso es la diferencia entre la PA sistólica y diastólica.

\section{DIAGNÓSTICO}

Tiene como propósito principal:

- Confirmar la elevación crónica de la PA y su nivel.

- Excluir causas secundarias y/o potencialmente curables de HTA.

- Determinar la presencia de daño en órgano blanco y cuantificar su magnitud.

- Buscar otros factores de riesgo cardiovascular y condiciones clínicas asociadas.

\section{MEDICIÓN DE LA PRESIÓN ARTERIAL $^{(1,2,4)}$}

Se señala en las próximas líneas indicaciones para optimizar la medición de la presión arterial.

- Medir la PA varias veces y en distintas ocasiones, con el paciente en posición sentada, con los pies apoyados al suelo y con algunos minutos de reposo ${ }^{(5)}$. No ingerir café ni fumar 30 minutos antes.

- Usar tensiómetro calibrado y validado.

- El brazo descubierto, en extensión y apoyado a la altura del corazón.
- En la primera visita, hacerlo en ambos brazos y considerar el mayor registro para utilizarlo en futuras mediciones.

- La insuflación debe ser hasta $30 \mathrm{mmHg}$ por encima del nivel de desaparición del pulso palpatorio.

- El tamaño del brazalete apropiado y colocado a $2 \mathrm{~cm}$ por encima del pliegue del codo.

- Realizar al menos dos mediciones separadas por 1 o 2 minutos.

- Medir la PA de pie ${ }^{(1-3)}$ en ancianos y diabéticos o ante sospecha de hipotensión ortostática.

Se debe registrar tanto la PAS como la PAD. La primera aparición de sonido (fase 1) se usa para definir la PAS. Su desaparición (fase 5) registra la PAD.

\section{AUTOMEDICIÓN DE LA PA (AMPA)}

La medición de la PA en casa beneficia a los pacientes, al proporcionar información sobre la respuesta de la medicación antihipertensiva, mejorando la adherencia al tratamiento ${ }^{(5)}$ y evaluando el efecto de la 'hipertensión de bata blanca'. Una PA mayor de 135/85 mmHg registrada en el hogar, generalmente es considerada como hipertensión ${ }^{(6)}$. 
Tabla 1. Clasificación de la presión arterial para adultos (18 años o mayores).

\begin{tabular}{lcc}
\hline Clasificación PA & PAS & PAD \\
& $\mathrm{mmHg}$ & $\mathrm{mmHg}$ \\
\hline Normal & $<120$ & $\mathrm{y}<80$ \\
Pre hipertensión & $120-139$ & $\mathrm{u} 80-89$ \\
Estadio 1 hipertensión & $140-159$ & $090-99$ \\
Estadio 2 hipertensión & $>160$ & $0>100$ \\
\hline
\end{tabular}

PA: presión arterial, PAS: presión arterial sistólica, PAD: presión arterial diastólica.

The JNC 7 Report, $2003^{(2)}$

Se recomienda los aparatos electrónicos validados o los esfigmomanómetros anaeroides ${ }^{(4)}$, cuya exactitud debe evaluarse periódicamente ${ }^{(7)}$.

\section{MONITORIZACIÓN AMBULATORIA DE LA PRESIÓN ARTERIAL (MAPA)}

Este procedimiento se efectúa mediante monitores especialmente programados para realizar lecturas cada quince a treinta minutos, brindando información de la PA durante las actividades diarias y durante el sueño nocturno. Su uso es de utilidad en la HTA resistente, en pacientes con síntomas de hipotensión bajo tratamiento, episodios hipertensivos, disfunción autonómica y registro de la PA durante las 24 horas, con miras a un mejor reajuste terapéutico ${ }^{(8)}$.

La PA ambulatoria es usualmente más baja que las lecturas obtenidas en la clínica (debajo de $135 / 85 \mathrm{mmHg}$ ) y los valores son menores aún durante el sueño (debajo de 120/75 mmHg). Usualmente, la PA disminuye $10 \%$ a $20 \%$ durante la noche y aquellos en quienes esto no ocurre (non-dipper) presentan mayor riesgo de eventos cardiovasculares.

Tabla 2. Causas identificables de hipertensión ${ }^{(2)}$.

- Apnea del sueño

- Inducción por drogas

- Enfermedad renal crónica

- Aldosteronismo primario

- Enfermedad renovascular

- Síndrome de Cushing

- Feocromocitoma

- Coartación de la aorta

- Enfermedad tiroidea o paratiroidea
El nivel de la PA mediante el uso de MAPA correlaciona mejor con el daño de órgano blanco ${ }^{(2,9)}$, que las mediciones en consultorio ${ }^{(9,10)}$.

\section{EVALUACIÓN CLÍNICA}

La evaluación clínica del paciente se realiza de la siguiente manera ${ }^{(11)}$ :

- Revisar la historia clínica y familiar.

- Interrogar sobre síntomas de daño en órgano blanco, estilos de vida e identificar los factores de riesgo cardiovascular y enfermedades concomitantes.

- Además de la correcta medición de la PA, palpar los pulsos arteriales centrales y periféricos, evaluar soplos en el cuello y abdominales, palpación de glándula tiroidea, examen de corazón y pulmones, examen de abdomen que incluya riñones, detección de tumoraciones y pulsaciones aórticas, verificar edema de miembros inferiores y valoración neurológica.

- Calcular el índice de masa corporal (peso (kg)/talla al cuadrado (m)); medida de la circunferencia de la cintura.

- Examen del fondo de ojo.

\section{EXÁMENES DE LABORATORIO}

Los análisis rutinarios incluyen un electrocardiograma (ECG), examen de orina (para sangre, glucosa, proteínas, sedimento), glicemia, hematocrito, potasio y calcio séricos, ácido úrico, creatinina (o filtrado glomerular), además de colesterol total, colesterol LDL y HDL, triglicéridos (tras 9 a 12 horas de ayuno) ${ }^{(2,10,12)}$.

El ECG permite la detección de hipertrofia ventricular izquierda, arritmias y enfermedad coronaria.
Se solicita pruebas complementarias en situaciones especiales: ecocardiograma, ultrasonido carotídeo y femoral, proteína C reactiva ultrasensible, microalbuminuria (esencial en diabéticos) ${ }^{(12)}$; se reserva pruebas específicas en la investigación de la hipertensión secundaria.

\section{FACTORES DE RIESGO VASCULAR (MAYORES)}

Los factores mayores de riesgo vascular son los siguientes: hipertensión arterial, tabaquismo, obesidad, sedentarismo, dislipidemia (hipercolesterolemia, hipertrigliceridemia y/o colesterol HDL bajo), diabetes mellitus, microalbuminuria o tasa de filtración glomerular estimada $<60 \mathrm{~mL} / \mathrm{min}$, edad (varones $>55$ años, mujeres $>65$ años), historia familiar de enfermedad cardiovascular prematura (varones $<55$ años, mujeres $<65$ años).

\section{LESIÓN EN ÓRGANO BLANCO ${ }^{(2)}$}

Las lesiones en órgano blanco pueden estar relacionadas con los siguientes problemas:

- Cardiaco: hipertrofia ventricular izquierda, angina o infarto miocárdico previo, revascularización coronaria previa, insuficiencia cardiaca.

- Cerebral: evento cerebrovascular o ataque isquémico transitorio.

- Enfermedad renal crónica.

- Vasculopatía periférica.

- Retinopatía.

- La estratificación del riesgo con fines de manejo y pronóstico se establece en base a los niveles de PA, factores de riesgo, daño de órgano blanco y condiciones clínicas asociadas.

\section{OBESIDAD Y SÍNDROME METABÓLICO}

El sobrepeso y la obesidad representan la causa ambiental más común de HTA. Su relación a las complicaciones cardiovasculares y renales es continua y progresiva, incluso estando la PA debajo del punto de quiebre tradicional, $140 / 90 \mathrm{mmHg}{ }^{(13)}$. 
El síndrome metabólico se define con 3 o más de las siguientes condiciones ${ }^{(14)}$ : obesidad abdominal (circunferencia $>90$ $\mathrm{cm}$ en hombres y $>80 \mathrm{~cm}$ en mujeres de origen latinoamericano) ${ }^{(15)}$, intolerancia a la glucosa (glucosa en ayunas $>100 \mathrm{mg}$ $\%)$, PA $>130 / 85 \mathrm{mmHg}$, triglicéridos $(>150 \mathrm{mg} / \mathrm{dL})$ o HDL $(<40 \mathrm{mg} / \mathrm{dL}$ en hombres o $<50 \mathrm{mg} / \mathrm{dL}$ en mujeres).

\section{ERRORES DE CONCEPTO FRECUENTES RELATIVOS A LA HIPERTENSIÓN ARTERIAL}

Los siguientes son errores de concepto frecuentes de los profesionales de la salud y de los pacientes, relacionados a la HTA:

- Se piensa que la PA sistólica aumenta con los años (añadiendo 100 a la edad).

- Que siempre se puede controlar la PA con cambios en los estilos de vida.

- Que sujetos con HTA deben limitar sus actividades.

- Se sigue estimando que la HTA diastólica es más importante.

- Que se puede suspender la medicación cuando la PA se encuentra bajo control.

- Que la HTA necesariamente debe ser sintomática.

La hipertensión arterial no solo debe definirse como un trastorno de la salud de cifras elevadas de la PA, sino como un síndrome que engloba alteraciones clínicas, hemodinámicas, tróficas y metabólicas ${ }^{(10)}$.

Igualmente, la conducta médica no debe basarse exclusivamente en los datos numéricos de la PA, sino considerar a su vez la presencia de otros factores de riesgo, enfermedades asociadas y lesiones de órgano blanco, sin menospreciar la importancia que conllevan los aspectos personales, socioeconómicos, demográficos, étnicos y culturales del paciente.

\section{REFERENCIAS BIBLIOGRÁFICAS}

1. The sixth report of the Joint National Committee on prevention, detection, evaluation, and treatment of high blood pressure. Arch Intern Med. 1997;157:2413-46.

2. Chobanian AV, Barris Gl, Black HR, Cushman WC, Green LA, Izzo JL Jr, Jones DW, Materson BJ, Oparil S, Wright JT Jr, Roccella EJ; Joint National Committee on Prevention, Detection, Evaluation, and Treatment of High Blood Pressure. National Heart, Lung, and Blood Institute; National High Blood Pressure Education Program Coordinating Committee. The Seventh Report of the Joint National Commitee on prevention, detection, evaluation, and treatment of high blood pressure: The JNC 7 Report. JAMA. 2003;289:2560-72.

3. Lewington S, Clarke R, Qizilbash N, Peto R, Collins $\mathrm{R}$; Prospective Studies Collaboration. Age-specific relevance of visual blood pressure to vascular mortality: A meta-analysis of individual data for one million adults in 61 prospective studies. Lancet. 2002;360:1903-13.

4. Prisant LM, Alfest BS. Robbins CB, Berson AS, Hayes M, Cohen ML, Sheps SG. American National Standard for non automated sphygmomanometers. Summary report. Am J Hypertens. 1995;8:210-3.

5. Appel LI, Staton WB. Ambulatory blood pressure monitoring and blood pressure self-measurement in the diagnosis and management of hypertension. Ann Intern Med. 1993;118:867-82.

6. Tsuji I, Imai Y, Nigai K, Ohkubo T, Watanabe N, Minami N, Itoh O, Bando T, Sakuma M, Fukao A, Satoh H, Hisamichi S, Abe K. Proposal of reference values for home blood pressure measurement prognostic criteria based on a prospective observation of the general population in Ohasanna, Japan. Am J Hypertens. 1997;10:409-18.

7. Consumer Reports. Blood-pressure monitors: convenience doesn't equal accuracy. Consumer Rep. 1996;61:50,53-55.

8. Pickering TG, Miller NH, Ogedegbe G, Krakoff LR, Artinian NT, Goff D; American Heart Association;
American Society of Hypertension; Preventive Cardiovascular Nurses Association. Call to action on use and reimbursement for home blood pressure monitoring: a joint scientific statement from the American Heart Association, American Society of Hypertension, and Preventive Cardiovascular Nurses Association. J Cardiovasc Nurs. 2008;23(4):299-323.

9. Verdecchia P. Prognostic value of ambulatory blood pressure: current evidence and clinical implications. Hypertension. 2000;35:844-51.

10. 1999 World Health Organization. International Society of Hypertension Guidelines for the Management of Hypertension. Guidelines Subcommittee. J Hypertens. 1999;17:151-83.

11. Victor RG, Kaplan NM. Systemic hypertension: mechanisms and diagnosis. En: Libby P, Bonow RO, Mann DL, Zifes DP, eds. Libby. Brauwald's Heart Disease: A Textbook of Cardiovascular Medicine, 8th ed. Saunders; 2007: chap 40.

12. European Society of Hypertension-European Society of Cardiology Guidelines Committee. 2003 European Society of HypertensionEuropean Society of Cardiology guidelines for the management of arterial hypertension. J Hypertens. 2003;21(6):1011-53.

13. World Hypertension League Journal Meeting. Hypertension/Obesity Project Prague, Czech Republic, June 22, 2002.

14. National Cholesterol Education Program (NCEP) Expert Panel on Detection, Evaluation, and Treatment of High Blood Cholesterol in Adults (Adult Treatment Panel III) final report. Third Report of the National Cholesterol Education Program (NCEP) Expert Panel on Detection, Evaluation, and Treatment of High Blood Cholesterol in Adults (Adult Treatment Panel III) final report. Circulation, 2002;106:3143-421.

15. Garcia RG, Cifuentes AE, Caballero RS, Sanchez L, López-Jaramillo P. A proposal for an appropriate central obesity diagnosis in Latin American population. Int J Cardiol. 2006;110:263-4.

\section{Correspondencia:}

Dr. Armando Vidalón Fernández

Av. Alonso de Molina 338, Valle Hermoso, Santiago

de Surco, Lima 3, Perú

Correo-e: armandovidalon@hotmail.com 